\title{
Diagnóstico de malaria por el método de la PCR anidada
}

\author{
Nohora M. Mendoza ${ }^{1}$, Carlos A. Jaramillo ${ }^{2}$, Felipe Guhl ${ }^{2}$, \\ Julio C. Padilla ${ }^{3}$, Martha C. Rentería ${ }^{4}$ \\ 1 Laboratorio de Parasitología, Instituto Nacional de Salud, Bogotá, D.C., Colombia. \\ 2 Centro de investigaciones en Microbiología y Parasitología Tropical, Universidad de los Andes, Bogotá, \\ D.C., Colombia. \\ ${ }^{3}$ División Técnica de Campañas Directas, Ministerio de Salud, Bogotá, D.C., Colombia. \\ ${ }^{4}$ Departamento Administrativo del Chocó, Quibdó, Colombia.
}

En 1998, se presentaron en Colombia 256.697 casos de malaria: 132.712 por Plasmodium falciparum, 121.161 por Plasmodium vivax y 2.824 infecciones mixtas. Dada esta situación, el Programa de Malaria del Laboratorio Nacional de Referencia se propuso evaluar la PCR anidada frente a la gota gruesa, como método alternativo para el diagnóstico de malaria.

Se utilizó la reacción en cadena de la polimerasa (PCR) anidada para amplificar secuencias variables de genes de la subunidad ribosomal pequeña 18 S (SsrARN) de $P$. falciparum y $P$. vivax. Para evaluar la PCR, se recolectaron 102 muestras de pacientes febriles en Quibdó (Chocó) y se determinó la presencia de parásitos tanto por PCR como por observación microscópica (gota gruesa). Los resultados de las dos pruebas se compararon entre sí.

Los criterios de evaluación fueron: precisión, exactitud, límite de detección, sensibilidad, especificidad, valores predictivos positivo (VPP) y negativo (VPN) e índice kappa.

La PCR anidada presentó una sensibilidad general del 100\%: 96,7\% para P.vivax y 100\% para $P$. falciparum. La especificidad general para $P$. vivax y para $P$. falciparum fue del $100 \%$. El VPP general de la prueba fue del $90 \%$ y el VPN fue de $90,3 \%$; para $P$. vivax, el VPP fue de $77,0 \%$ y el VPN de 90,6\%; para P. falciparum, el VPP fue de 66,1\% y el VPN de 94,3\%. El índice kappa general fue de 1 y el de especie fue de 0,96. El límite de detección del ADN parasitario fue de 10 $\mathrm{fg} / \mathrm{ml}$. La PCR anidada detectó una muestra con infección mixta de $P$. vivax y $P$. falciparum que, por gota gruesa, dio resultado positivo para $P$. vivax.

Se recomienda el uso de la PCR anidada como método de tamizaje en estudios epidemiológicos para la evaluación del Programa de Control Nacional de Malaria, en estudios orientados a detectar de manera temprana parasitemias en pacientes tratados cuya posible causa pueda ser la resistencia a los antimaláricos y como prueba de referencia para evaluar nuevos métodos diagnósticos.

Palabras clave: PCR, diagnóstico, malaria.

\section{Malaria diagnosis by nested PCR}

In 1998, 256,697 malaria cases were confirmed in Colombia, 132,712 of which were caused by Plasmodium falciparum, 121, 161 by, and 2,824 mixed infections. Consequently, the Malaria Program of the National Reference Laboratory initiated an evaluation of the nested polymerase chain reaction (PCR) technique for malaria diagnosis. This technique amplifies variable sequences present in the 18S small subunit ribosomal RNA (ssrRNA) genes of $P$. falciparum and $P$. vivax. The infected blood samples were collected in Quibdó (Chocó) from 102 febrile patients who requested malaria diagnosis; the presence of parasites was established by both PCR and microscopical examination of thick blood smears. In order to evaluate the nested PCR, the following criteria were adopted: precision, accuracy, detection treshhold, sensitivity, specificity, positive and negative predictive values and Kappa index. General sensitivity was $100 \%$, with a sensitivity for $P$. vivax of $96.7 \%$ and for $P$. falciparum of $100 \%$. General specificity and specificity for both species were $100 \%$. The general positive predictive value was $90.0 \%$, for $P$. vivax $77.0 \%$ and for $P$. falciparum $66.1 \%$. The general negative predictive value was $90.3 \%, 90.6 \%$ for $P$. vivax and $94.3 \%$ for $P$. falciparum. The general concordance (or Kappa index) was 1 and the species concordance 
was 0.96 . The detection treshhold of parasite DNA was $10 \mathrm{fg} / \mu \mathrm{l}$. One sample diagnosed as $P$. vivax by microscopy was determined as a mixed infection of $P$. falciparum and $P$. vivax by nested PCR. Nested PCR can be employed as a diagnostic tool in the following roles: (1) a screening method for large scale epidemiological trials, (2) in evaluations of the malaria control program in Colombia, (3) in detection of early or low levels of persistent parasitemia, possibly indicative of drug-resistant malaria, and (4) as a reference test for planned diagnostic trials.

Key words: PCR, diagnosis, malaria.

En 1998 se registraron en Colombia 256.697 casos de malaria, para una tasa de $6,5 / 1.000$ hab en las zonas de riesgo. La distribución por especie fue de $52 \%$ para Plasmodium falciparum, $47 \%$ para Plasmodium vivax y $1 \%$ para infecciones mixtas. Los departamentos con mayor número de casos, en su orden, fueron: Chocó, Córdoba, Antioquia, Valle, Guaviare, Meta y Nariño (1).

En la actualidad, existen varias alternativas para el diagnóstico de malaria: ParaSight- ${ }^{\star}$, OptiMal ${ }^{\circledast}$ ICT Malaria Pf, QBC, coloración de naranja de acridina con o sin el sistema de filtros de interferencia y técnicas de biología molecular; sin embargo, la prueba de referencia continúa siendo la gota gruesa. En el examen microscópico de la gota gruesa es posible realizar la identificación de las especies y el recuento parasitológico. El Plasmodium en la gota gruesa puede ser detectado en bajas densidades parasitarias, del orden de 20 a 30 parásitos/ $\mu$ l de sangre (2), incluso, se reporta que es posible realizar su detección a partir de 10 parásitos/ $\mu$ l de sangre (3). La gota gruesa tiene una sensibilidad superior al $80 \%$ y una especificidad del $76 \%$ (4). Los anteriores parámetros dependen de la experiencia del lector. Por otra parte, el nivel de detección parasitaria puede afectarse por una coloración inadecuada, un microscopio con óptica deficiente o la fatiga ocular del microscopista debida a la observación de un alto volumen de muestras. Por otra parte, el diagnóstico de malaria se complementa con el extendido, que tiene un nivel de detección entre 100-200 parásitos/ $\mu \mathrm{i}$.

Entre las técnicas de biología molecular se han desarrollado pruebas de PCR específicas tanto para $P$. falciparum $(5,6)$ como para $P$. vivax $(7,8)$.

Correspondencia:

Nohora Marcela Mendoza

mmendoza@hemagogus.ins.gov.co

Recibido: 16/01/01; aceptado: 14/09/01
También se han utilizado métodos basados en la amplificación de las secuencias variables de los genes que codifican para la subunidad ribosomal pequeña, con los cuales es posible obtener diagnósticos específicos para las cuatro especies de Plasmodium que ocasionan la malaria humana (9-11).

Para una máxima sensibilidad y especificidad, se emplea la PCR anidada, en donde el producto de la primera reacción (Nest-1) se usa como plantilla o molde de ADN para la segunda amplificación (Nest-2), en la cual los iniciadores empleados reconocen secuencias contenidas dentro del producto de ADN de la reacción Nest-1. Las ventajas de este método son el aumento de la sensibilidad en la detección al obtenerse un mayor número de copias del fragmento amplificado y disminuir la cantidad de ADN molde. Las desventajas, al compararla con la PCR no añidada, son el requerir más tiempo y materiales, como también el mayor riesgo de contaminación. Esta última desventaja se puede evitar siguiendo las normas preventivas de contaminación para la PCR. Por otra parte, la ventaja de la PCR anidada frente a la gota gruesa es su capacidad de detectar parasitemias submicroscópicas. La desventaja es la de no poderse emplear como un método diagnóstico de rutina por no tener la capacidad de cuantificar la parasitemia y el ser técnicamente más compleja, demorada, dispendiosa y costosa (12).

La situación epidemiológica de la malaria en Colombia se ha visto agravada por problemas como los desplazamientos de poblaciones, la intensificación del conflicto social armado, la demanda laboral de cultivos ilícitos, la poca participación social, la eliminación de los grupos operativos de control de vectores, la falta de compromiso político para solucionar el problema, la baja capacidad de la gestión técnica y operativa 
del nivel departamental y municipal, la falta de compromiso para la ejecución de las acciones operativas, la mala utilización de los recursos, las malas prácticas políticas y la centralización del programa de malaria por parte de algunos departamentos (Ministerio de Salud, reunión nacional de evaluación de los programas de enfermedades transmitidas por vectores, Bogotá, 2000).

Para suministrar una herramienta que fortalezca técnicamente el programa de malaria en el abordaje de los problemas epidemiológicos a gran escala, en la evaluación de nuevos métodos de diagnóstico de malaria (pruebas rápidas de diagnóstico de campo) y en la confirmación de casos clínicos difíciles o especiales, se probó una PCR anidada para el diagnóstico de malaria en Quibdó, Chocó.

\section{Materiales y métodos}

El lugar del estudio fue Quibdó, Chocó. Quibdó es la cabecera municipal del departamento y está constituida por una planicie muy húmeda con abundante precipitación durante 10 meses del año. La altura sobre el nivel del mar es de $32 \mathrm{~m}$; la temperatura media es de $26,4^{\circ} \mathrm{C}$ y la precipitación media anual corresponde a 7,748 mm (13). En este municipio se dispusieron tres sitios para la captación de pacientes: el Centro de Salud San Vicente de Paúl, el Instituto de los Seguros Sociales y el Hospital de San Francisco de Asís. La toma de muestras para realizar el diagnóstico de gota gruesa y la PCR anidada se realizó entre diciembre de 1998 y febrero de 1999.

El tipo de estudio fue descriptivo de corte transversal.

Se incluyeron en el estudio todos aquellos pacientes que cumplieron con la definición de caso probable de malaria que solicitaron el diagnóstico de malaria en uno de los tres puntos de toma de muestras y que, además, consintieron por escrito en suministrar $5 \mathrm{ml}$ de sangre total en tubo al vacio con EDTA para efectuar el diagnóstico de la PCR anidada. Un caso probable de malaria se define como toda persona residente o procedente de zona endémica que presenta malaria con fiebre, escalofrío, sudor y mialgias o cefalea.

Se excluyó todo paciente menor de 7 años o mayor de 80 años, con evidencia de alguna complicación por malaria (malaria cerebral, colapso circulatorio, anemia severa, hemorragias, falla renal, convulsiones, edema pulmonar, ictericia, hipoglicemia, hiperparasitemia, hiperpirexia o hemoglobinuria), manifestación de parálisis parcial o total y presencia de adenopatías.

Se determinó una muestra de 76 pacientes probables casos de malaria usando un muestreo de corte transversal y teniendo en cuenta la eficiencia del método ( $97 \%$ ) con un error del $5 \%$.

A todos los pacientes se les practicó una gota gruesa para diagnóstico de malaria $y$, adicionalmente, se obtuvieron $5 \mathrm{ml}$ de sangre total anticoagulada con EDTA, con la cual se realizaron dos láminas de gota gruesa, una para hacer el diagnóstico por personal especializado y otra para efectuar el control de calidad. La muestra de sangre total también se utilizó para efectuar la extracción de ADN para la técnica de PCR anidada. El remanente se separó en alícuotas y se almacenó a $-20{ }^{\circ} \mathrm{C}$. Las muestras se identificaron con el número consecutivo de toma de muestras de cada puesto. La toma de la muestra de sangre se hizo previa solicitud de consentimiento informado a los pacientes que se incluyeron en el estudio, según los lineamientos establecidos en la Resolución No. 08430 de 1993 del Ministerio de Salud. Todos los pacientes incluidos en el estudio recibieron el tratamiento específico recomendado por el Ministerio de Salud de acuerdo con el resultado del examen de gota gruesa. Este proyecto fue presentado, discutido y aprobado en todos sus aspectos por el Instituto Nacional de Salud, el Ministerio de Salud y la Dirección Departamental de Salud del Chocó.

La prueba diagnóstica de oro fue la gota gruesa coloreada con la tinción de Romanowsky, modificada en el Laboratorio Departamental de Salud Pública del Chocó y en el Instituto Nacional de Salud, con protocolos estandarizados y siguiendo los lineamientos de control de calidad del Instituto Nacional de Salud. La parasitemia se calculó con el recuento de las formas parasitarias frente a 100 leucocitos y se empleó como constante leucocitaria 8.000 leucocitos $/ \mathrm{mm}^{3}(14)$.

La extracción y purificación de ADN para la PCR anidada se realizó con el estuche comercial de 
Wizard $^{\mathrm{TM}}$ Genomic DNA a partir de $100 \mu \mathrm{l}$ de la sangre total anticoagulada con EDTA. En general, el sistema de extracción ocurre en cuatro etapas: en el primer paso sucede la lisis celular que deja libres los núcleos, los cuales se lisan en el segundo paso; posteriormente, en la tercera etapa, ocurre la precipitación proteica con sales, y en el cuarto paso, una precipitación del ADN con isopropanol.

La primera lisis del protocolo de extracción de ADN se modificó ya que previamente se habia realizado lisis celular con saponina como parte de otro protocolo de extracción de ADN con el cual se obtuvieron buenos resultados. Aun tubo Eppendorf nuevo de $1,5 \mathrm{ml}$ se le adicionó la muestra de sangre total y 7 volúmenes de saponina al $0,15 \%$. La saponina se disolvió en solución salina, fosfato isotónico tamponada (PBS isotónico). Se incubó a $37^{\circ} \mathrm{C}$ durante 30 minutos. Se centrifugó a 13.000 rpm durante 30 minutos y se efectuaron tres lavados, cada uno con $400 \mu \mathrm{l}$ de PBS isotónico. Después de resuspender suavemente el precipitado con pipeta, se continuó con la lisis de núcleos según el protocolo del estuche de extracción de ADN.

Para la primera amplificación correspondiente al género Plasmodium, se emplearon los iniciadores rPLU5 y rPLU6. En la segunda amplificación, se trabajaron iniciadores específicos para especie; se emplearon los oligonucleótidos rFAL1 y rFAL2 para identificar $P$. falciparum y rV1V1 y rV1V2 para $P$. vivax (9). Los volúmenes y las concentraciones de los reactivos por muestra procesada se presentan en el cuadro 1 y los programas de amplificación utilizados en el cuadro 2. Los amplificados tuvieron un tamaño de 205 pb y 120 $\mathrm{pb}$, respectivamente. Los productos de amplificación se visualizaron en geles al 3\% usando agarosa NuSieve ${ }^{\circledR}$ y Seakem ${ }^{\circledR}$ en proporcion 2:1.

Se determinó la exactitud, la precisión, el límite de detección, la sensibilidad, la especificidad, el índice kappa y los valores predictivos de la prueba (15-17).

Se trabajó con la exactitud clínica y experimental en la etapa de la estandarización de la técnica. La exactitud clínica consistió en visualizar el amplificado de ADN según la especie infectante de Plasmodium a partir de las muestras de sangre total con el resultado conocido de la gota gruesa. En la exactitud experimental, se trabajó por duplicado con diluciones seriadas de ADN de concentración conocida de $P$. falciparum para observar la relación existente entre la concentración del ADN y la intensidad de la banda en el gel.

La reproducibilidad del método se realizó trabajando quintuplicados de tres muestras con el procedimiento estandarizado.

Para establecer la mínima concentración de ADN parasitario detectable por la PCR anidada, se diluyó una muestra de ADN de P. falciparum y de $P$. vivax. La concentración inicial de la dilución fue $1 \mathrm{ng} / \mu \mathrm{l}$ de sangre.

La información obtenida se almacenó y analizó en una base de datos de Epilnfo 6.04.

Cuadro 1. Concentraciones y volúmenes recomendados por muestra para la amplificación 1 en la prueba de la PCR anidada.

\begin{tabular}{lrrr}
\hline Reactivo & Concentración inicial & Concentración final & Volumen por muestra de reacción $(\boldsymbol{\mu})$ ) \\
\hline Tampón & $10 \mathrm{~m}$ & $1 \mathrm{X}$ & 2,5 \\
MgCl $_{2}$ & $25 \mathrm{mM}$ & $1 \mathrm{mM}$ & 1,0 \\
dNTPS & $5 \mathrm{mM}$ & $0,125 \mathrm{mM}$ & 0,6 \\
rPLU5 & $2.500 \mathrm{nM}$ & $250 \mathrm{nM}$ & 2,5 \\
rPLU6 & $2.500 \mathrm{nM}$ & $250 \mathrm{nM}$ & 2,5 \\
Agua destilada & - & - & 14,8 \\
ADN & - & - & 1,0 \\
Taq polimerasa & $5 \mathrm{U}$ & $0,4 \mathrm{U}$ & 0,1 \\
\hline
\end{tabular}

Para trabajar la segunda amplificación, se tuvieron en cuenta las mismas concentraciones incluso para adicionar los iniciadores de especie (rFAL1, rFaL2 y rV1V1, rV1V2). Se hizo el montaje en vial independiente para cada pareja de iniciadores. 
Cuadro 2. Diagnóstico de malaria por la prueba de la PCR anidada; programas de amplificación, amplificación 1.

\begin{tabular}{lcc}
\hline Pasos & Temperatura ${ }^{\circ} \mathrm{C}$ & Tiempo (min) \\
\hline Inicio de la desnaturación & 95 & 5 \\
Anillado & 58 & 2 \\
Extensión & 72 & 2 \\
Desnaturación & 94 & 1 \\
Se repiten los pasos 2-4 por un total de 25 veces & 58 & 2 \\
Anillado final & 72 & 5 \\
Extensión final & 4 & \\
Finalización de la reacción & & \\
\hline
\end{tabular}

El programa de amplificación de la segunda reacción difiere de la primera solamente en el número de ciclos, pues se repiten los pasos 2-4 por un total de 30 veces.

\section{Resultados}

Se estudiaron 102 pacientes, 61 hombres y 41 mujeres. 65 pacientes acudieron al Centro de Salud San Vicente de Paúl, 27 al Hospital San Francisco de Asís y 10 al Instituto de los Seguros Sociales. El $83 \%$ de los pacientes que acudieron para diagnóstico de malaria tenían entre 7 y 36 años. La positividad general de la población examinada fue de $53 \%$. De los 102 pacientes examinados, 52 fueron positivos y 50 negativos para malaria utilizando los métodos de gota gruesa y la PCR anidada (cuadro 3 ).

La PCR anidada mostró una sensibilidad general del $100 \%, 96,7 \%$ para $P$. vivax y $100 \%$ para $P$. falciparum. La especificidad general para $P$. vivax y $P$. falciparum fue del $100 \%$. El índice kappa general fue 1 y el de especie 0,96 . Por otra parte, el VPP general fue de $90,0 \%, 77,0 \%$ para $P$. vivax y $66,1 \%$ para $P$. falciparum, mientras que el VPN general fue de $90,3 \%, 90,6 \%$ para $P$. vivax y $94,3 \%$ para $P$. falciparum. Sin embargo, los valores predictivos aplicados a la población con la prevalencia de malaria para 1998 fueron inferiores (cuadro 4). El límite de detección de ADN parasitario fue $10 \mathrm{fg} / \mu \mathrm{l}$.

Los resultados de la PCR anidada tanto en la etapa de estandarización como en la etapa del montaje de las muestras del estudio fueron concordantes con los resultados obtenidos por gota gruesa y, así, se pudo verificar la exactitud clínica y al trabajar la exactitud experimental, se observó en el gel que la intensidad de la banda disminuyó a medida que descendió la concentración de la dilución de ADN. Por otra parte, la técnica fue reproducible
Cuadro 3. Diagnóstico para malaria por microscopia y PCR anidada.

\begin{tabular}{lccccc}
\hline $\begin{array}{l}\text { Pruebas } \\
\text { diagnósticas }\end{array}$ & \multicolumn{4}{c}{ Microscopía } & $\begin{array}{c}\text { Total } \\
\text { examinados }\end{array}$ \\
\cline { 2 - 5 } & Positivos & Negativos & \\
PCR anidada & PV & Pf & Total & & \\
Positivas & $32^{*}$ & 20 & 52 & 0 & 52 \\
Negativas & 0 & 0 & 0 & 50 & 50 \\
Total & 32 & 20 & 52 & 50 & 102 \\
\hline
\end{tabular}

* Una muestra diagnosticada por gota gruesa como positiva para $P$. vivax se diagnosticó como infección mixta $(P$. vivax y $P$ falciparum) por PCR anidada.

Pv: P. vivax; Pf: $P$. falciparum.

bajo las condiciones de trabajo estandarizadas, con lo cual se determinó la precisión.

Los amplificados obtenidos por la PCR anidada tuvieron los tamaños esperados, que correspondieron a $205 \mathrm{pb}$ y $120 \mathrm{pb}$ y que identificaron a $P$. falciparum y $P$. vivax, respectivamente (figura 1).

\section{Discusión}

Los resultados indican que los valores de la sensibilidad y especificidad de este estudio fueron superiores, aunque cercanos a los reportados por otros autores $(8,12,18,19)$, lo cual podría explicarse porque la técnica original de extracción de ADN fenol-cloroformo (10) fue sustituida por un sistema de extracción metodológicamente más sencillo y rápido, con el cual es posible obtener ADN genómico de cadena doble a partir de sangre total humana procedente de glóbulos blancos y de eucariotes que se encuentren en circulación sanguínea $(20,21)$. El método consta de una extracción inorgánica o digestión con proteinasas. Por otra parte, los iniciadores.utilizados son empleados para obtener un diagnóstico especie - 
Cuadro 4. Sensibilidad, especificidad y valores predictivos de la PCR anidada para el diagnóstico de malaria.

\begin{tabular}{lccccc}
\hline & Sensibilidad (\%) & Especificidad (\%) & Prevalencia* (\%) & VPP (\%) & VPN\% \\
\hline General & 100 & 100 & 50,0 & 90,0 & 90,3 \\
Valor IC 95\% & $91,4-100$ & $91,1-100$ & 14,5 & 60,5 & 98,2 \\
P. vivax & 96,7 & 100 & 30,0 & 77,0 & 90,6 \\
Valor IC 95\% & $82,0-99,8$ & $80,0-100$ & 3,3 & 10,6 & 98,9 \\
P. falciparum & 100 & 100 & 20,0 & 66,1 & 94,3 \\
Valor IC 95\% & $80,0-100$ & $86,7-100$ & 10,5 & 37,5 & 97,2 \\
\hline
\end{tabular}

VPP: valor predictivo positivo, VPN: valor predictivo negativo, IC 95\%: intervalo de confianza del 95\%; P: prevalencia *: se calcularon los VPP y VPN teniendo en cuenta tanto la prevalencia de malaria en la población estudiada (50\%) como las prevalencias de malaria en general y por cada una de las especies en el departamento del Chocó para 1998.

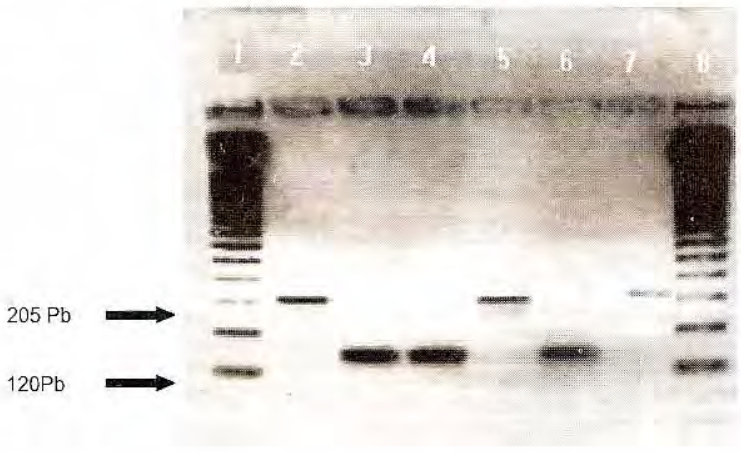

Figura 1. Electroforesis en gel de agarosa de amplificados de muestras obtenidas de pacientes positivos para malaria. Carriles: 1 y 8 : patrón de peso molecular 50 pb DNA Step Ladder $®$ (Promega); 2: control positivo de $P$. falciparum (cepa FcB2: Falciparum Colombia Bogotá 2); 3: control positivo de $P$. vivax (CV: control vivax); 4: muestra 85: $P$. vivax; 5: muestra 157: $P$. falciparum; 6 y 7: muestra 123: infección mixta ( $P$ vivax y $P$. falciparum).

específico. A pesar de que en la literatura se describe que el ADN humano en grandes cantidades altera la eficiencia de la reacción, al actuar como inhibidor (10), en el presente estudio no se observó dicho efecto.

Solamente se encontró una muestra discordante entre los dos métodos con la gota gruesa positiva para $P$. vivax y positiva para infección mixta por la PCR anidada. Este resultado fue reproducible en varios ensayos y puede tener dos explicaciones. La primera es que la PCR anidada pudo haber detectado una parasitemia submicroscópica, hallazgo observado en otros trabajos $(8,10,12,20,22)$, lo cual puede atribuirse a que el límite de detección de la prueba fue de10 $\mathrm{fg} / \mu \mathrm{l}$ de ADN, es decir, menos de la cantidad de $A D N$ que tiene un solo parásito, razón por la cual es altamente probable encontrar este tipo de resultado. La segunda es la posibilidad de una eventual contaminación en el procedimiento de extracción de ADN. Sin embargo, esta posibilidad es poco probable porque con las alícuotas de sangre total de la misma muestra almacenadas a $-20^{\circ} \mathrm{C}$ se realizó una nueva extracción de ADN que arrojó los mismos resultados y porque los resultados obtenidos con todos los controles necesarios incluidos para detectar una eventual contaminación fueron siempre los esperados.

Cuando sucede una infección mixta, existe un período en el que se presenta antagonismo entre las dos especies de Plasmodium, lo cual ocasiona que una de ellas predomine sobre la otra (10). Por otra parte, el diagnóstico de las infecciones mixtas tiene mayor dificultad en las zonas endémicas en donde es posible encontrar pacientes con infecciones latentes o subpatentes, o cuando se presenta una baja parasitemia en la cual morfológicamente predominan las formas anulares (11). Tienen especial interés los casos de infección mixta en los cuales $P$. falciparum se encuentra enmascarado por $P$. vivax, como sucedió en este caso, debido a que la infección puede conducir al paciente a un desenlace fatal, más aún, considerando que en Colombia circulan cepas resistentes a los medicamentos antimaláricos (23-28).

Las características de la PCR anidada permiten que esta prueba pueda ayudar a aclarar algunos interrogantes de tipo epidemiológico para entender mejor la dinámica de transmisión de la malaria en 
un foco determinado. Por ejemplo, es útil para demostrar bajos niveles de transmisión o nuevos focos en lugares en donde se consideraba controlada la malaria $(19,29)$. Además, puede ser adecuada para evaluar la proporción de casos de infecciones mixtas, considerada como un indicador del programa de control cuyo aumento sugiere un deterioro.

La PCR ofrece la posibilidad de ser aplicada en estudios especiales que determinen la negativización parasitaria postratamiento y así detectar precozmente posibles fallas terapéuticas, pues se ha demostrado que esta prueba arroja resultados positivos siempre que el parásito esté viable. Cuando los parásitos han muerto o han sido eliminados por el tratamiento o por presión del sistema inmune, no se observa producto de amplificado por la PCR $(19,30)$. También puede ser útil en el seguimiento de pacientes inmunizados en estudios para evaluar la eficacia de posibles vacunas antimaláricas. Cuando se adelanten estudios en los cuales sea necesario procesar un gran número de muestras, se recomienda contar con dos o más laboratoristas para hacer menos dispendioso el procedimiento y obtener los resultados más rápidamente. La PCR anidada también se puede aplicar para evaluar otros métodos de diagnóstico, teniendo en cuenta que esta metodología permite determinar falsos positivos y negativos en la gota gruesa (10), como también problemas en la identificación de la especie. Por las anteriores razones, se considera que la PCR anidada es una buena alternativa que el Laboratorio Nacional de Referencia de Parasitología y otros laboratorios y centros de investigación pueden emplear en estudios especiales y de tipo epidemiológico dirigidos, por ejemplo, para establecer las prevalencias reales y la dinámica de la transmisión en áreas donde ocurren parasitemias submicróscópicas, no detectables por los métodos usuales que, aunque no originan usualmente sintomatología en los pacientes, sí permiten la persistencia de la transmisión (29).

Aunque la gota gruesa sigue siendo la prueba de oro en el diagnóstico de malaria, su capacidad de detección es 40 veces menor lo cual constituye una limitación frente a la PCR (20). Por este motivo, durante la ejecución de este trabajo se esperaba encontrar algunas muestras negativas por gota gruesa y positivas por PCR $(10,11)$, lo cual no sucedió debido, muy posiblemente, a que solamente se incluyeron pacientes sintomáticos en quienes es muy alta la probabilidad de encontrar niveles de parasitemia circulante detectables por gota gruesa. Si se hiciera un estudio para buscar la presencia de la parasitemia circulante en población asintomática de áreas endémicas sería más probable encontrar personas negativas por gota gruesa y positivas por PCR anidada.

Por su facilidad, rapidez y bajo costo, la gota gruesa sigue siendo el método de primera elección para confirmar por el laboratorio el diagnóstico de malaria, especialmente en zonas remotas que cuentan con recursos mínimos de laboratorio. Por el contrario, la PCR anidada, por ser una metodología dispendiosa, costosa, que requiere de equipos e infraestructura complejas, no se recomienda para el diagnóstico y seguimiento de rutina del paciente con malaria y su aplicación se justifica solamente en centros de referencia cuando se realizan estudios especiales como los mencionados anteriormente.

\section{Agradecimientos}

Los autores expresan sus agradecimientos a Rubén Santiago Nicholls por la revisión y corrección del documento, a Sofía Duque por la corrección del manuscrito y a Fernando de la $\mathrm{Hoz}$ y Alexandra Porras por su amable colaboración en el análisis estadístico.

\section{Referencias}

1. Ministerio de Salud. Informe ejecutivo semanal. Sistema Nacional de Vigilancia en Salud Pública 1999; Semana epidemiológica $\mathrm{N}^{\circ} 20$ (16 a 22 de mayo,1999).

2. Cox-Singh J, Mahayet S, Abdullah MS, Singh B. Increased sensitivity of malaria detection by nested polymerase chain reaction using simple sampling and DNA extraction. Int J Parasitol 1997;27:1575-7.

3. Castelli F, Carosi G. Diagnosis of malaria infection. En: Carlosi G, Castelli F, editores. Handbook of malaria infection in the tropics. Bologna: Editorial Board; 1997. p. 114.

4. Clendennen III TE, Long GW, Baird JK. QBC and Giemsa-stained thick blood films: diagnostic performance of laboratory technologists. Trans R Soc Trop Med Hyg • 1995;89:183-4. 
5. Arai M, Mizuroshi $\mathrm{CH}$, Kubochi F, Kakutani T, Wataya Y. Detection of Plasmodium falciparum in human blood by nested polymerase chain reaction. Am J Trop Med Hyg 1994;51:617-26.

6. Barker RH, Banchongaksorn $T$, Couva! JM, Suwonkerd W, Rimwungtragoon K, Wirth DF. A simple method to detect Plasmodium falciparum from blood samples using the polymerase chain reaction. Am J Trop Med Hyg 1992;46:416-26.

7. Del Portillo HA, Parrillo IB, Camargo AA, Levitt A. Amplification of single-copy genes of Plasmodium vivax from two drops of peripheral blood of infected patients. J Protozool Res 1993;3:20-4.

8. Tirasophon $\mathbf{W}$, Rajkulchai $\mathbf{P}$, Ponglikitmongkol $\mathbf{M}$, Wilairat $P$, Boonsaeng V, Panyim S. A highly sensitive, rapid, and simple polymerase chain reaction-based method to detect human malaria (Plasmodium falciparum and Plasmodium vivax) in blood samples. Am J Trop Med Hyg 1994;51:308-13.

9. Snounou G, Viriyakosol S, Zhu XP, Jarra W, Pinheiro Y, Rosario VE, et al. High sensitivity of detection of human malaria parasites by the use of nested polymerase chain reaction. Mol Biochem Parasitol 1993;61:315-20.

10. Snounou G, Viriyakosol S, Jarra W, Thaithong S, Brown N. Identification of the four human malaria parasite species in field samples by the polymerase chain reaction and detection of a high prevalence of mixed infections. Mol Biochem Parasitol 1993;58:283-92.

11. Snounou G, Pinheiro I, Goncalves A, Fonseca L, Dias F, Brown KN, et al. The importance of sensitive detection of malaria parasites in the human and insect hosts in epidemiological studies, as shown by the analysis of field samples from Guinea Bissau. Trans $R$ Soc Trop Med Hyg 1993;87:649-53.

12. Singh B, Cox-Singht J, Miller AO, Abdullah MS, Snounou G, Rahman HA. Detection of malaria in Malaysia by nested polymerase chain reaction amplification of dried blood spots on filter papers. Trans R Soc Trop Med Hyg 1996;90:519-21.

13. Instituto Geográfico Agustín Codazzi. Diccionario geográfico de Colombia. Santafé de Bogotá: Horizonte Impresores Ltda.; 1996. p. 1842.

14. Shute GT. The microscopic diagnosis of malaria. En: Wernsforfer $\mathrm{H}, \mathrm{Mc}$ Gregor IA, editors. Malaria: principles and practices of malariology. London: Longman Group UK, Limited; 1988. p. 781-813.

15. Riegelman RK, Hirsh RP. Cómo estudiar un estudio y probar una prueba: lectura crítica de la literatura médica. Publicación científica No. 531. Washington, D.C.: Organización Panamericana de la Salud; 1992.

16. Asociación Química Colombiana. Good laboratory practice: validación de sistemas analíticos. III. Noticias Químicas 1999;20:20.
17. Guerrero R, González CL, Medina E. Epidemiología. Wilmington: Addison-Wesley Iberoamericana, S.A; 1986. p. $174-77$.

18. Weiss JB. DNA probes and PCR for diagnosis of parasitic infections. Clin Microbiol Rev 1995;8:113-30.

19. Laserson KF, Petralanda I, Hamlin DM, Almera R, Fuentes M, Carrasquel A, et al. Use of the polymerase chain reaction to directly detect malaria parasites in blood samples from the Venezuelan Amazon. Am J Trop Med Hyg 1994;50:169-80.

20. Cox-Singh J, Mahayet S, Abdullah MS, Singh B. Increased sensitivity of malaria detection by rested polymerase chain reaction using simple sampling and DNA extraction. Int J Parasitol 1997;27:1575-7.

21. Da Silva AJ, Bornay-Llinares FJ, Moura IN, Slemenda SB, Tuttle JL, Pieniazek NJ. Fast and reliable extraction of protozoan parasite DNA from fecal specimens. Mol Diagn 1999;4:57-64.

22. Brown AE, Krain KC, Pipithkul J, Kyle H. Demostration by the polymerase chain reaction of mixed Plasmodium falciparum and P.vivax infections undetected by conventional microscopy. Trans R Soc Trop Med Hyg 1992;86:609-12.

23. Restrepo A, Alvarez L, Restrepo M. Estudio in vivo de la resistencia del $P$. falciparum a la cloroquina en Colombia (descripción de la resistencia III). Acta Méd Colomb 1980;5:367-79.

24. Espinal CA, Uribe LM, Eslava A, Rodriguez ME. Resistencia del Plasmodium falciparum a la combinación sulfa-pirimetamina (descripción de los tres primeros casos). Biomédica 1981;1:213-17.

25. Espinal C, Moreno E, Guerra P, De La Vega P. Aislamiento y caracterización de cepas colombianas de Plasmodium falciparum. Biomédica 1982;2:118-28.

26. Espinal CA, Cortés GT, Guerra P, Arias AE. Sensitivity of Plasmodium falciparum to antimalarial drugs in Colombia. Am J Trop Med Hyg 1985;34:675-80.

27. Blair S. Resistencia del P. falciparum a drogas en Colombia 1961-1986. Biomédica 1986;6:95-100.

28. Payne D. Spread of chloroquine resistance inPlasmodium falciparum. Parasitol Today 1987;3:241246.

29. Roper C, Elhassan IM, Hviid L, Giha H, Richardson $\mathbf{W}$, Babiker $\mathrm{H}$, et al. Detection of very low level Plasmodium falciparum infections using the nested polymerase chain reaction and a reassessment of the epidemiology of unstable malaria in Sudan. Am J Trop Med Hyg 1996;54:325-31.

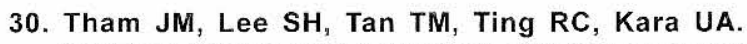
Detection and species determination of malaria parasites by PCR: comparison with microscopy and with Parasight-F and ICT malaria Pf tests in a clinical environment. J Clin Microbiol 1999;37:1269-73. 